\title{
Prognostic factors, treatment, and outcomes in combined small cell lung cancer: a SEER survey from 2004 to 2015
}

\author{
Long Xu, Guanzhong Zhang, Shuxi Song, Zhendong Zheng \\ Department of Oncology, General Hospital of Northern Theater Command, Shenyang, China \\ Contributions: (I) Conception and design: Z Zheng; (II) Administrative support: Z Zheng; (III) Provision of study materials or patients: L Xu; (IV) \\ Collection and assembly of data: L Xu, G Zhang; (V) Data analysis and interpretation: L Xu, S Song; (VI) Manuscript writing: All authors; (VII) \\ Final approval of manuscript: All authors. \\ Correspondence to: Zhendong Zheng. Department of Oncology, General Hospital of Northern Theater Command, 83 Wenhua Road, Shenhe District, \\ Shenyang 110016, China. Email: mylonzzdong2018@163.com.
}

\begin{abstract}
Background: The epidemiological characteristics of combined small cell lung cancer (C-SCLC) are still unclear. Therefore, in this study, we aimed to investigate the prognostic factors and treatment modalities for C-SCLC.

Methods: The clinical data from patients with primary C-SCLC from 2004 to 2015 were extracted from the Surveillance, Epidemiology, and End Results (SEER) database. Next, we applied the 8th edition of the TNM classification. A Cox regression model was used to identify the relevant variables and treatment modalities which affected the overall survival (OS) and cancer-specific survival (CSS).
\end{abstract}

Results: In total, 1,010 patients were included in the analysis. The median OS and median CSS were 9 months and 10 months, respectively. Individuals aged 65 and younger, female, and classified as being in an earlier stage were independent predictors of an improved OS and CSS. For patients with stage I, surgery alone significantly improved the OS (HR, 0.371; 95\% CI, 0.180-0.769) and CSS (HR, 0.367; 95\% CI, $0.162-0.892)$. For patients with stage II, surgery combined with radiotherapy significantly improved the OS (HR, 0.237; 95\% CI, 0.063-0.890). For patients with stage III, radiotherapy alone was an independent predictor of OS (HR, 0.464; 95\% CI, 0.316-0.618) and CSS (HR, 0.452; 95\% CI, 0.305-0.670). For patients with stage IV, surgery combined with radiotherapy significantly improved OS (HR, 0.315; 95\% CI, 0.181-0.547) and CSS (HR, 0.329; 95\% CI, 0.189-0.573), and surgery alone also improved OS (HR, 0.257; 95\% CI, 0.144-0.461) and CSS (HR, 0.280; 95\% CI, 0.156-0.501). Undergoing chemotherapy was also an independent predictor of an improved OS (HR, 0.619; 95\% CI, 0.419-0.915) in patients with stage III, and OS (HR, 0.283; 95\% CI, 0.228-0.350) and CSS (HR, 0.289; 95\% CI, 0.232-0.259) of patients with stage IV.

Conclusions: These findings may help to determine possible treatment choices and aid further research on this rare disease.

Keywords: Combined small cell lung cancer (C-SCLC); prognostic factors; treatment; cancer survival

Submitted Feb 11, 2020. Accepted for publication Jul 17, 2020.

doi: $10.21037 /$ tcr-20-968

View this article at: http://dx.doi.org/10.21037/tcr-20-968

\section{Introduction}

Combined small cell lung cancer (C-SCLC) was confirmed as a subset of small cell lung cancer (SCLC) by the WHO in 1999. C-SCLC is defined as pure SCLC combined with components of non-small cell lung cancer (NSCLC).
Recent studies have reported that C-SCLC accounts for $5 \%$ to $61 \%$ of all SCLC cases, and there is a nonhomogenous survival difference between pure SCLC and C-SCLC groups (1-3). In those earlier small-sample retrospective studies, the differences in these results may be attributed to the type of specimens used, and different 
population groups. Furthermore, other studies have shown that C-SCLC and pure SCLC have similar general characteristics of $(3,4)$. However, mixed-component NSCLC has been shown to have divergent genotypes compared with pure SCLC and to influence the prognosis $(3,5-7)$. To date, the prognostic factors of C-SCLC remain unclear.

The multidisciplinary treatment for C-SCLC is conducted according to the clinical practice SCLC guidelines. Further research on the optimized treatment modalities for C-SCLC is still necessary. Therefore, we aimed to report on a sample with a larger population, based on the data from the Surveillance, Epidemiology, and End Results (SEER) database [2004-2015]. We present the following article in accordance with the STROBE reporting checklist (available at http://dx.doi.org/10.21037/tcr-20-968).

\section{Methods}

\section{Patients}

The SEER database of the National Cancer Institute (NCI) covers $34.6 \%$ of the US population. In this study, the SEER-18 registry (with custom treatment fields) was explored using the official software, version 8.3.5. The data from patients with C-SCLC between the dates of 2004 and 2015 were extracted. C-SCLC was defined by the site recode ICD-O-3/WHO 2008 of the "lung and bronchus," and histologic type codes 8045 . The 8 th edition of the TNM classification was used. The exclusion criteria were: (I) unknown survival time; (II) cases without a positive pathological diagnosis; or (III) multiple primary cancers.

The following information was extracted: age at diagnosis, race, sex, TNM stages, tumor size and extension, primary site, grade, surgery, radiotherapy or chemotherapy, survival, vital status, and the causespecific death classification. We obtained internet access to the SEER database with the reference number 12025-Nov2017. As this study used a national dataset of deidentified patient information, it did not need to be approved by the institutional review board of the General Hospital of Northern Theater Command. The data of this observational study was anonymous; therefore, the requirement for informed consent was waived.

\section{Statistical analyses}

Continuous variables were reported as mean $\pm \mathrm{SD}$, and the categorical variables were reported as $\mathrm{n}(\%)$. Incidence rates per 100,000 age-adjusted to the 2000 US standard population and the annual percent change (APC) were calculated from 2004 to 2015 using SEER*Stat. Survival rates were analyzed using the Kaplan-Meier method and the log-rank test. The Cox regression model was used to identify the relevant variables and treatment modalities which affected the overall survival (OS) and cancer-specific survival (CSS). A two-sided $\mathrm{P}$ value $<0.05$ was considered statistically significant. Statistical analyses were performed using the SPSS software version 23.0 (SPSS Inc. Chicago, IL, USA), and the scatter diagrams were drawn using the GraphPad Prism 5.01 (GraphPad Software, San Diego, CA, USA).

\section{Results}

\section{Incidence rates and trends}

Between 2004 and 2015, the age-adjusted incidence rates for C-SCLC were substantially lower than pure-SCLC alone, with a rate of 0.15 per 100,000 for C-SCLC $(95 \%$ CI, 0.14-0.16) compared to a rate of 7.31 per 100,000 for pure-SCLC alone (95\% CI, 7.22-7.40). Over time, the incidence rates of pure-SCLC varied but had a significant overall decreasing trend (Figure 1A). The annual percentage of change for pure-SCLC alone was $-2.5 \%$ (95\% CI, -2.9 to $-2.1, \mathrm{P}<0.05)$. However, during the same period, the incidence rates for C-SCLC did not statistically decrease (Figure 1B). The annual percentage of change for C-SCLC was $-0.7 \%$ ( $95 \% \mathrm{CI},-3.1$ to $1.7, \mathrm{P}=0.5$ ).

\section{Epidemiological characteristics}

The SEER database included 1,378 patients with C-SCLC diagnosed from 2004 to 2015. Of these, we excluded 354 who were multiple primary cancer, 11 who were not pathologically diagnosed and 3 whose survival time were missed. A total of 1,010 patients were included in the analysis. The majority of patients were older than 65 years $(57.3 \%)$, males $(56.5 \%)$, and white $(83.8 \%)$. About half of the tumors with known sizes were $\leq 5 \mathrm{~cm}(52.2 \%)$. The upper lobe was the most common primary site (53.6\%). Almost half of the tumors were poorly differentiated (32.2\%) or undifferentiated $(16.1 \%)$. The numbers of patients in the cohort with stage I, II, III, and IV disease, were 142 (14.1\%), 68 (6.7\%), 257 (25.4\%), and 498 (49.3\%) respectively. Data were available on the local treatment modality for $98.8 \%$ 
A

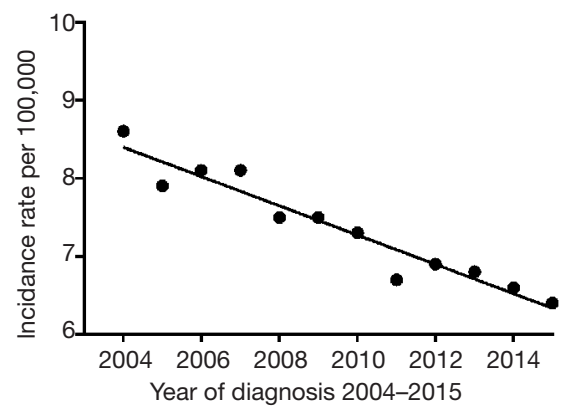

B

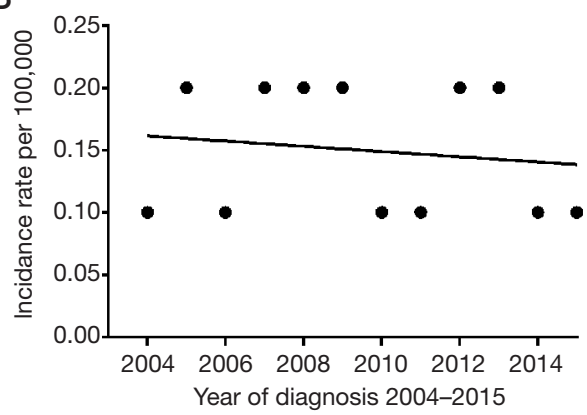

Figure 1 Age-adjusted incidence rates for small cell lung cancer. (A) Age-adjusted incidence rates for pure-small cell lung cancer by year of diagnosis from 2004 to 2015; (B) Age-adjusted incidence rates for combined small cell lung cancer by year of diagnosis from 2004 to 2015.

of patients, and chemotherapy was used on $100 \%$ of the patients (Table 1).

Kaplan-Meier analysis revealed that the median OS and median CSS for the entire cohort were 9 and 10 months, respectively. A younger age, being female, an early stage, and tumors that were highly differentiated, smaller than $5 \mathrm{~cm}$, or located in the upper lobe, were independent prognostic factors of C-SCLC; however, race did not correlate with OS and CSS (Figure 2, Table 1).

\section{Univariate and multivariate analysis}

As shown in Table 2 and Table 3, the Cox proportional hazard analyses were performed to investigate the independent prognostic factors of C-SCLC. In the unadjusted univariate analysis, the individual's age, sex, tumor size, stage, primary site, local treatment modality, and chemotherapy usage were significantly associated with the OS and CSS. Multivariate analyses were further adjusted by age, sex, stage, primary site, local treatment modality, and chemotherapy status. The results of multivariate analysis identified that being younger than 65 years, female, and at an earlier stage were all independent predictors of an improved OS and CSS, while the primary site was not. Compared to no local therapy, surgery combined with radiotherapy was significantly associated with an improved OS (HR, 0.456; 95\% CI, 0.336-0.618; $\mathrm{P}<0.001)$ and CSS (HR, 0.465; 95\% CI, 0.339-0.638; $\mathrm{P}<0.001)$. Surgery alone was also significantly associated with an improved OS (HR, 0.396; 95\% CI, 0.304-0.516; $\mathrm{P}<0.001)$ and CSS (HR, 0.393; 95\% CI, 0.296-0.521; $\mathrm{P}<0.001$ ). However, the use of radiotherapy alone was significantly associated with an improved OS (HR, 0.840; 95\% CI, 0.713-0.990; $\mathrm{P}=0.038$ ), but was not significantly associated with CSS (HR,
0.857; 95\% CI, 0.724-1.015; $\mathrm{P}=0.074)$. Chemotherapy also significantly improved the OS (HR, 0.384; 95\% CI, 0.328$0.451 ; \mathrm{P}<0.001)$ and CSS (HR, 0.385; 95\% CI, 0.327-0.455; $\mathrm{P}<0.001)$.

\section{Effect of therapy modality on TNM stage related-survival}

Kaplan-Meier survival analyses and Cox proportional hazard analyses were conducted to evaluate the effect of therapy modalities on the TNM stage related-survival (Tables 4,5). Multivariate analyses were then further adjusted by age, sex, local treatment modality, and chemotherapy. For stage I patients, surgery alone was the most common local therapy modality $(65.5 \%)$, and it was the only local therapy modality that had significantly improved the OS (HR, 0.371; 95\% CI, 0.180-0.769; $\mathrm{P}=0.008$ ) and CSS (HR, $0.367 ; 95 \%$ CI, $0.162-0.892 ; \mathrm{P}=0.016)$. Nearly half of the stage I patients (47.9\%) underwent chemotherapy, but the multivariate analysis revealed that it was not an independent prognostic factor.

In this cohort, $19(27.9 \%)$ patients with stage II underwent surgery combined with radiotherapy. Multivariate analyses showed that surgery combined with radiotherapy significantly improved the OS (HR, 0.237; 95\% CI 0.063-0.890; $\mathrm{P}=0.033$ ) but not CSS. Most patients (72.1\%) underwent chemotherapy, but the multivariate analysis revealed that it was not an independent prognostic factor.

Radiotherapy was the most common local therapy modality $(47.5 \%)$ for patients with stage III, and it was an independent predictor of the OS (HR, 0.464; $95 \%$ CI, 0.316-0.618; $\mathrm{P}<0.001)$ and CSS (HR, 0.452; 95\% CI, $0.305-0.670 ; \mathrm{P}<0.001)$. Multivariate analyses also showed that surgery combined with radiotherapy and surgery alone 
Table 1 Patient characteristics and survival analysis (month)

\begin{tabular}{|c|c|c|c|c|c|}
\hline Characteristic & No. (\%) & mOS $(95 \% \mathrm{Cl})$ & $P$ value & mCSS $(95 \% \mathrm{Cl})$ & $P$ value \\
\hline Age, years & & & $<0.001$ & & $<0.001$ \\
\hline$\leq 65$ & $431(42.7)$ & $11(9.5-12.5)$ & & $11(9.4-12.6)$ & \\
\hline$>65$ & 579 (57.3) & $8(6.7-9.3)$ & & $9(7.6-12.4)$ & \\
\hline Male & $571(56.5)$ & $8(6.8-9.2)$ & & $8(6.7-9.3)$ & \\
\hline Female & $439(43.5)$ & $12(10.4-13.6)$ & & $12.9(10.2-13.8)$ & \\
\hline Race & & & 0.716 & & 0.726 \\
\hline White & $846(83.8)$ & $9.0(8.0-10.0)$ & & $10.0(9.0-11.0)$ & \\
\hline Tumor grade & & & $<0.001$ & & $<0.001$ \\
\hline Highly differentiated & $12(1.2)$ & $26.0(3.4-48.7)$ & & $26(3.4-48.6)$ & \\
\hline Moderately differentiated & $36(3.6)$ & $12.0(6.6-17.4)$ & & $12(6.6-17.4)$ & \\
\hline Poorly differentiated & $325(32.2)$ & $11.0(8.2-13.8)$ & & $12(9.2-14.8)$ & \\
\hline Undifferentiated & $163(16.1)$ & $10.0(7.2-12.8)$ & & $11(8.1-13.9)$ & \\
\hline Unknown & $474(46.9)$ & $7.0(5.8-8.2)$ & & $8(6.7-9.3)$ & \\
\hline Tumor size & & & $<0.001$ & & $<0.001$ \\
\hline I & $142(14.1)$ & $47.0(18.2-75.8)$ & & $78.0(46.1-109.9)$ & \\
\hline II & $68(6.7)$ & $21.0(11.0-31.0)$ & & $21.0(10.2-31.8)$ & \\
\hline III & $257(25.4)$ & $13.0(10.6-15.4)$ & & $14.0(11.3-16.7)$ & \\
\hline IV & $498(49.3)$ & $5.0(4.1-5.9)$ & & $5.0(4.1-5.9)$ & \\
\hline Unknown & $45(4.5)$ & $6.0(2.7-9.3)$ & & $6.0(3.4-8.6)$ & \\
\hline Primary site & & & $<0.001$ & & $<0.001$ \\
\hline Main bronchus & $72(7.1)$ & $6(2.6-9.4)$ & & $8(4.9-11.1)$ & \\
\hline Upper lobe & $541(53.6)$ & $11(9.6-12.4)$ & & $11(9.1-12.9)$ & \\
\hline Middle lobe (right lung only) & $38(3.8)$ & $8(1.0-15.0)$ & & $8(1.0-15.0)$ & \\
\hline Lower lobe & $230(22.8)$ & $8(6.0-10.0)$ & & $9(7.0-11.0)$ & \\
\hline Overlapping lesion of lung & $18(1.8)$ & $9(0.0-19.3)$ & & $10.0(0.0-20.3)$ & \\
\hline Unknown & $111(11.0)$ & $4(1.3-6.7)$ & & $5.0(2.4-7.6)$ & \\
\hline
\end{tabular}

Table 1 (continued) 
Table 1 (continued)

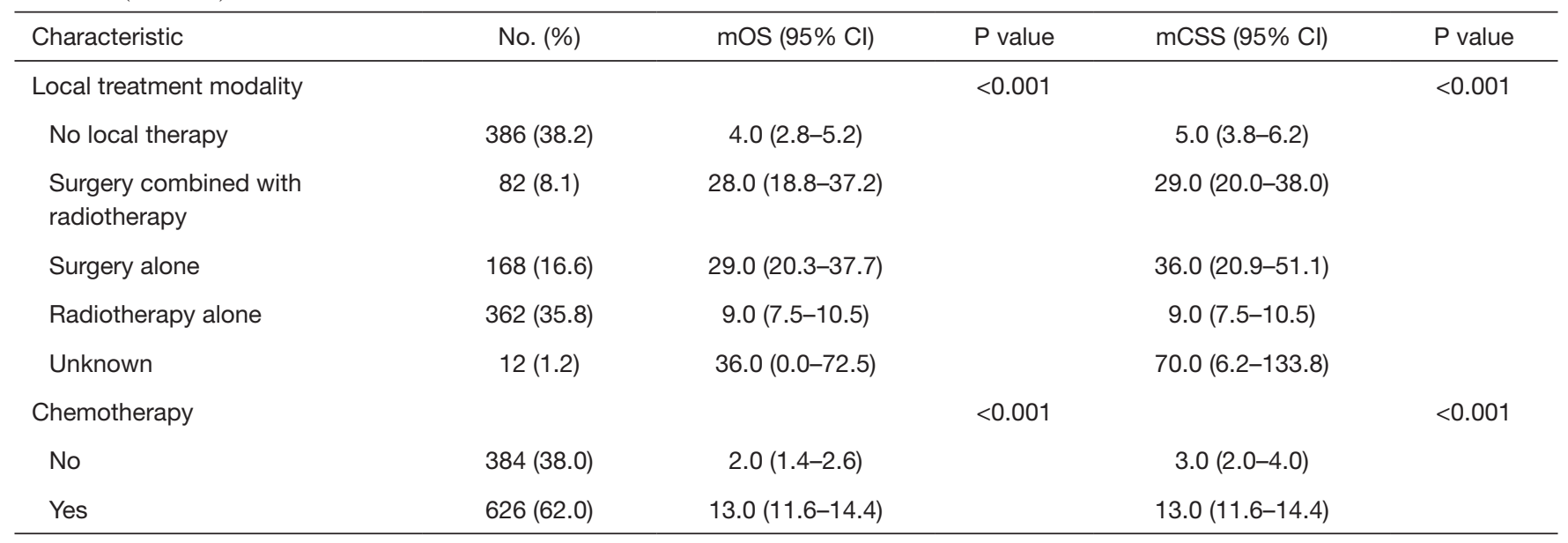

Undefined indicates that median survival time was incalculable due to death occurring in less than $50 \%$ of the cases in the cohort.

could result in an improved OS and CSS. However, 31.9\% of patients did not undergo any local treatment modality. Finally, chemotherapy was an independent predictor of an improved OS (HR, 0.619; 95\% CI, 0.419-0.915; $\mathrm{P}=0.016$ ) but not CSS.

Surgery was rarely performed on patients with stage IV (4.0\% for surgery combined with radiotherapy, and 3.2\% for surgery alone); however, it was determined that it was associated with improved survival. The median OS was 22 months for patients who underwent surgery combined with radiotherapy, 10 months for patients who underwent surgery alone, and only 3 months for patients with no local therapy. Multivariate analyses revealed that surgery combined with radiotherapy significantly improved the OS (HR, 0.315; 95\% CI, 0.181-0.547) and CSS (HR, 0.329; $95 \%$ CI, 0.189-0.573), and surgery alone also improved the OS (HR, 0.257; 95\% CI, 0.144-0.461) and CSS (HR, 0.280; $95 \%$ CI, 0.156-0.501). Of the stage IV patients, $40.6 \%$ underwent radiotherapy alone as a local therapy; however, it was not an independent predictor of the OS (HR, 1.071; 95\% CI, 0.877-1.307; $\mathrm{P}=0.501)$ and CSS (HR, 1.094; 95\% CI, 0.893-1.340; $\mathrm{P}=0.386$ ). However, chemotherapy was an independent predictor of an improved OS (HR, 0.283; 95\% CI, 0.228-0.350; $\mathrm{P}<0.001)$ and CSS $(\mathrm{HR}, 0.289 ; 95 \% \mathrm{CI}$, $0.232-0.259$; $\mathrm{P}<0.001)$; meanwhile, $41.2 \%$ of patients with stage IV did not undergo chemotherapy.

\section{Discussion}

C-SCLC is a rare subtype of SCLC. The majority of patients with SCLC are heavy smokers, and so the decrease in the overall amount of smokers may account for the decrease in the incidence rate of pure-SCLC (8). However, our data showed that the incidence of C-SCLC remained stable between 2004 and 2015, while the incidence of pureSCLC decreased. This may be explained by advances in diagnostic and surgical techniques; therefore, C-SCLC could be detected more easily.

Many scientists have devoted time and effort to understanding the mechanisms and histogenesis of C-SCLC (9-11). Zhao et al. recently reported on a next generation sequencing (NGS) technique; they identified that the histologic components of C-SCLC had an overall consistency and only partial differences (3). Asahina et al. described a case of C-SCLC with squamous-like component (SLC), and spindle cell carcinomas (SpCC) components. In the study, it was demonstrated that by analyzing histological, immunohistochemical, and loss of heterozygosity (LOH) events, it was determined that the components of a nonsmall cell carcinoma were not differentiated from a small cell carcinoma (12); however, further studies are still needed.

Earlier studies found there to be some similar prognostic factors for C-SCLC patients. A retrospective review of 97 individuals with resected C-SCLC identified that sex, age, ECOG PS, NSCLC components, adjuvant therapy were significant prognostic factors that influenced the OS (1). Another study of 114 patients with resected C-SCLC showed that smoking, advanced TNM stage, positive resection margin, and lymph node metastasis $>10 \%$ were poor prognostic factors (4). Our multivariate analysis also confirmed that sex, age, and stage were independent 

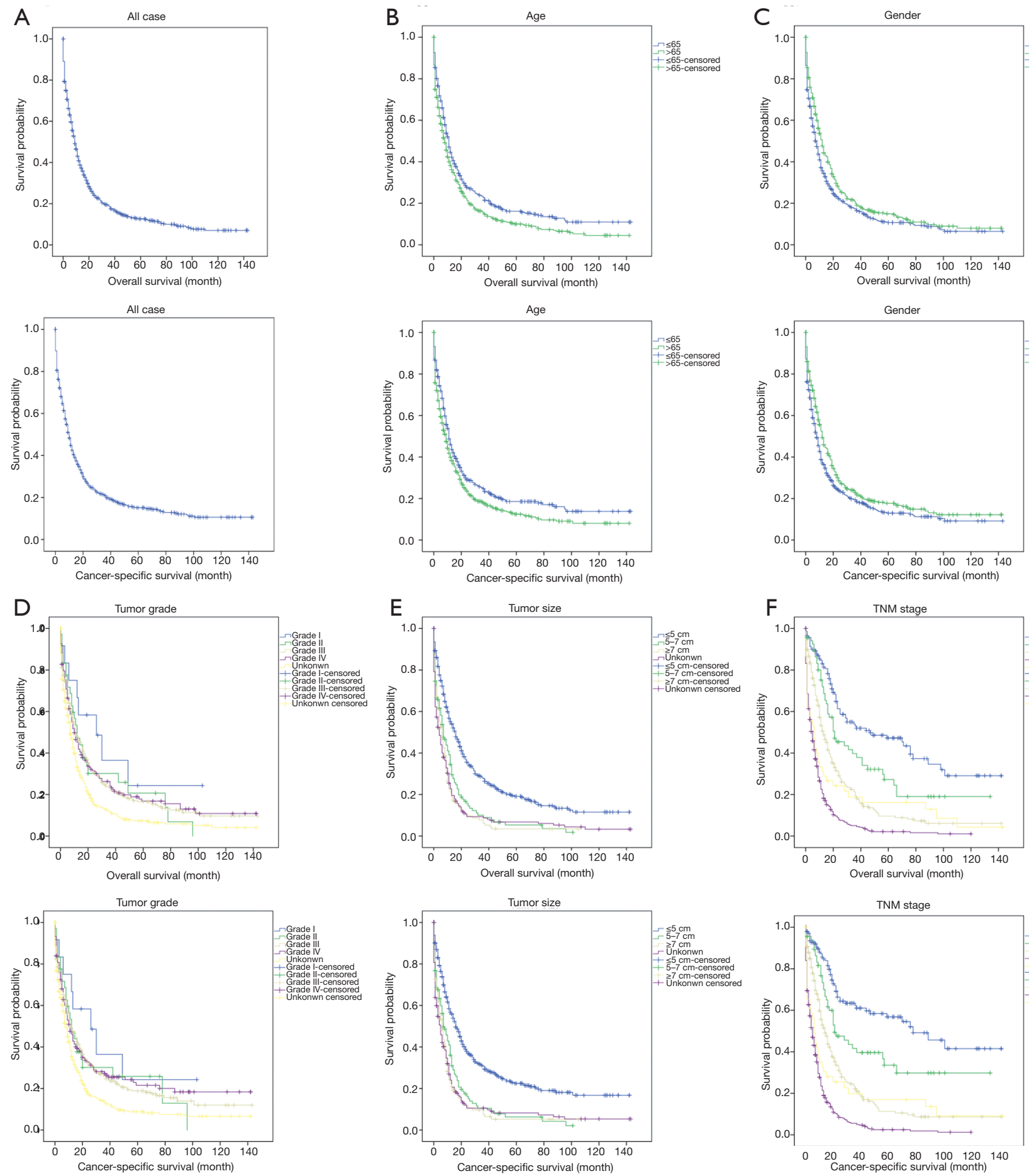

Figure 2 Kaplan-Meier curves for overall survival and cancer-specific survival. (A) In all cases; (B) age; (C) gender; (D) tumor grade; (E) tumor size; (F) tumor, node, metastasis stage. 
Table 2 Univariate Analysis of overall survival and cancer-specific survival

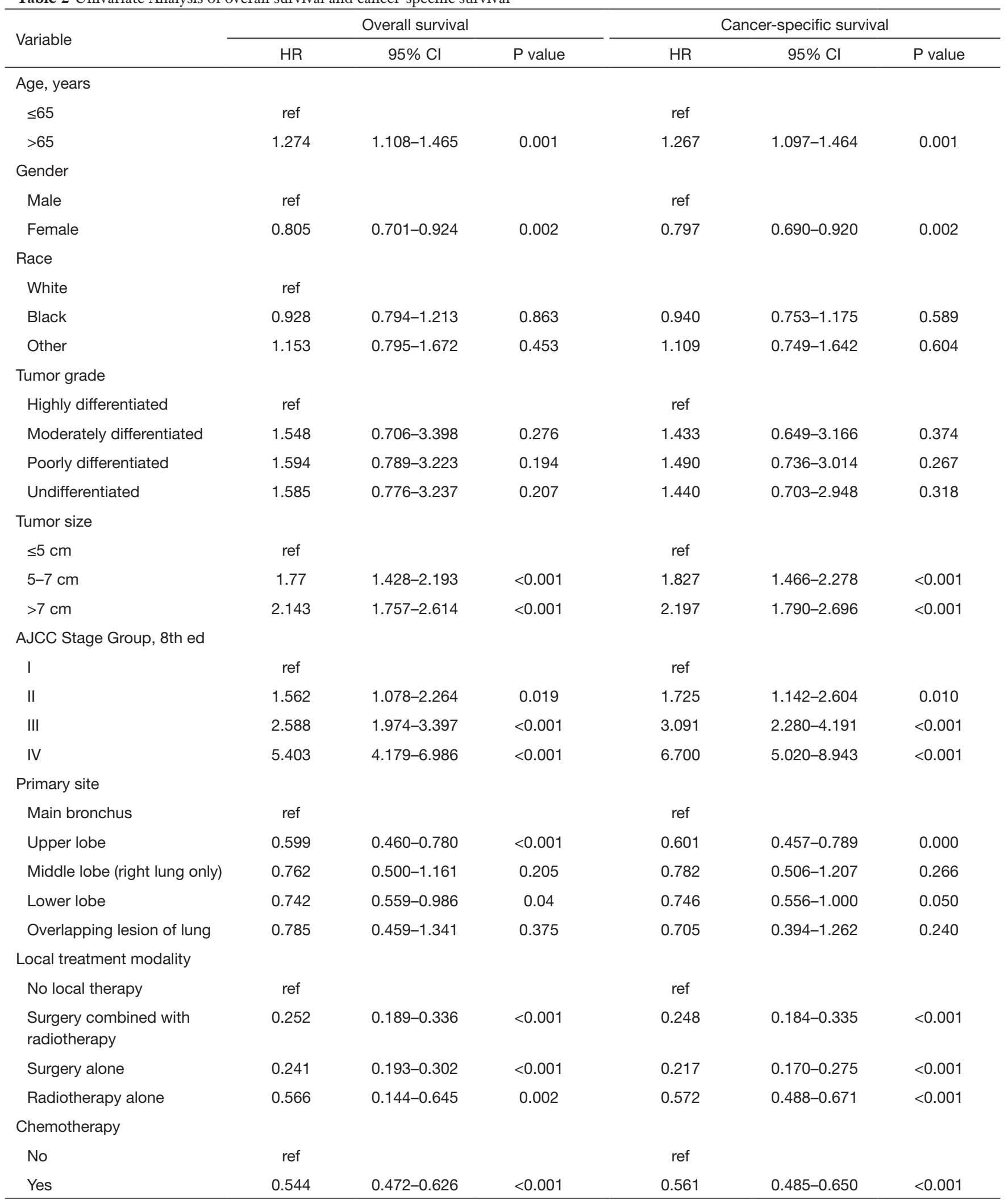


Table 3 Multivariate analysis of overall survival and cancer-specific survival

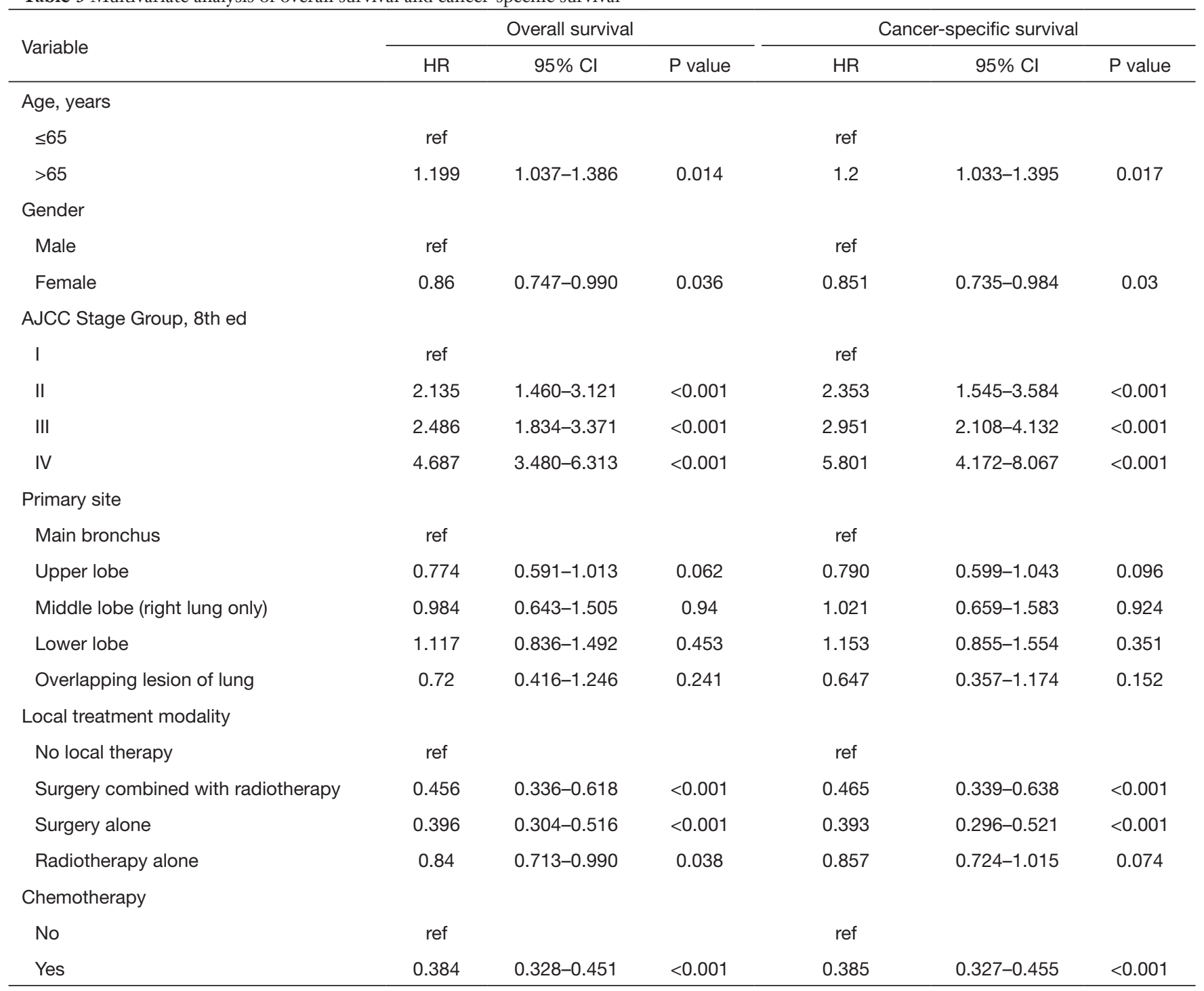

prognostic factors. The main difference was that the previous studies above only focused on postoperative C-SCLC, while our study included patients of any stage who had and had not undergone surgery. Additionally, we also analyzed surgery combined with radiotherapy and surgery alone as local therapy modalities. We found that they had significantly improved the OS and CSS, and radiotherapy alone significantly improved the OS, but not the CSS. Chemotherapy was found as a significant prognostic factor for determining OS and CSS. As a result, multidisciplinary treatment was recommended to improve the prognosis. Other studies also showed that neutrophilelymphocyte ratio (NLR) and C-reactive protein (CRP) amounts were associated with prognosis in C-SCLC (13-15). These results could be applied to clinical management as a reference and are deserving of further study.

By using the TNM staging system, clinicians can identify patients who may benefit from surgery. Surgical treatment was recommended only for SCLC patients with a T1-2N0M0 stage according to the NCCN guidelines (16). However, in our present study, surgery was identified as a critical factor in improving the prognosis of individuals with C-SCLC, especially for stage I patients, most of whom had undergone surgery. Furthermore, surgery alone or surgery combined with radiotherapy was important local therapy for patients with stage II and III. Survival time was significantly 


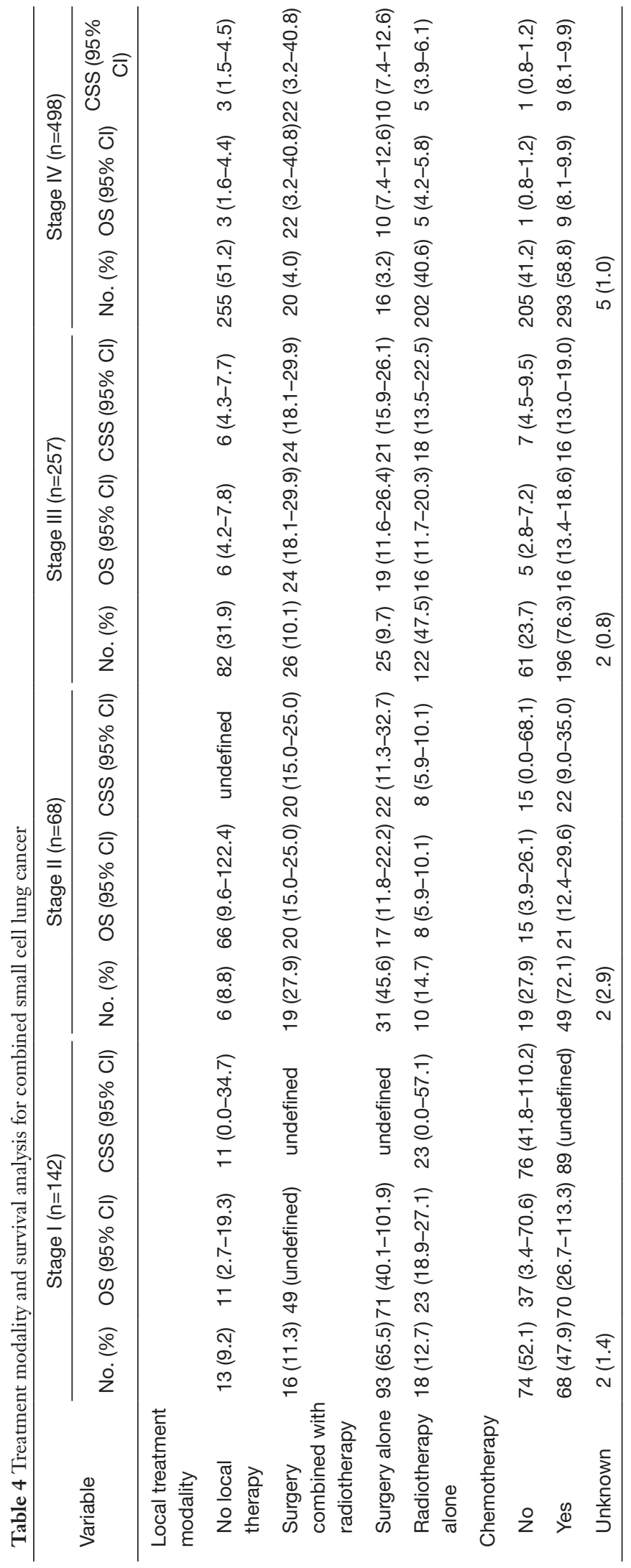


Table 5 Multivariate analysis of treatment for overall survival and cancer-specific survival

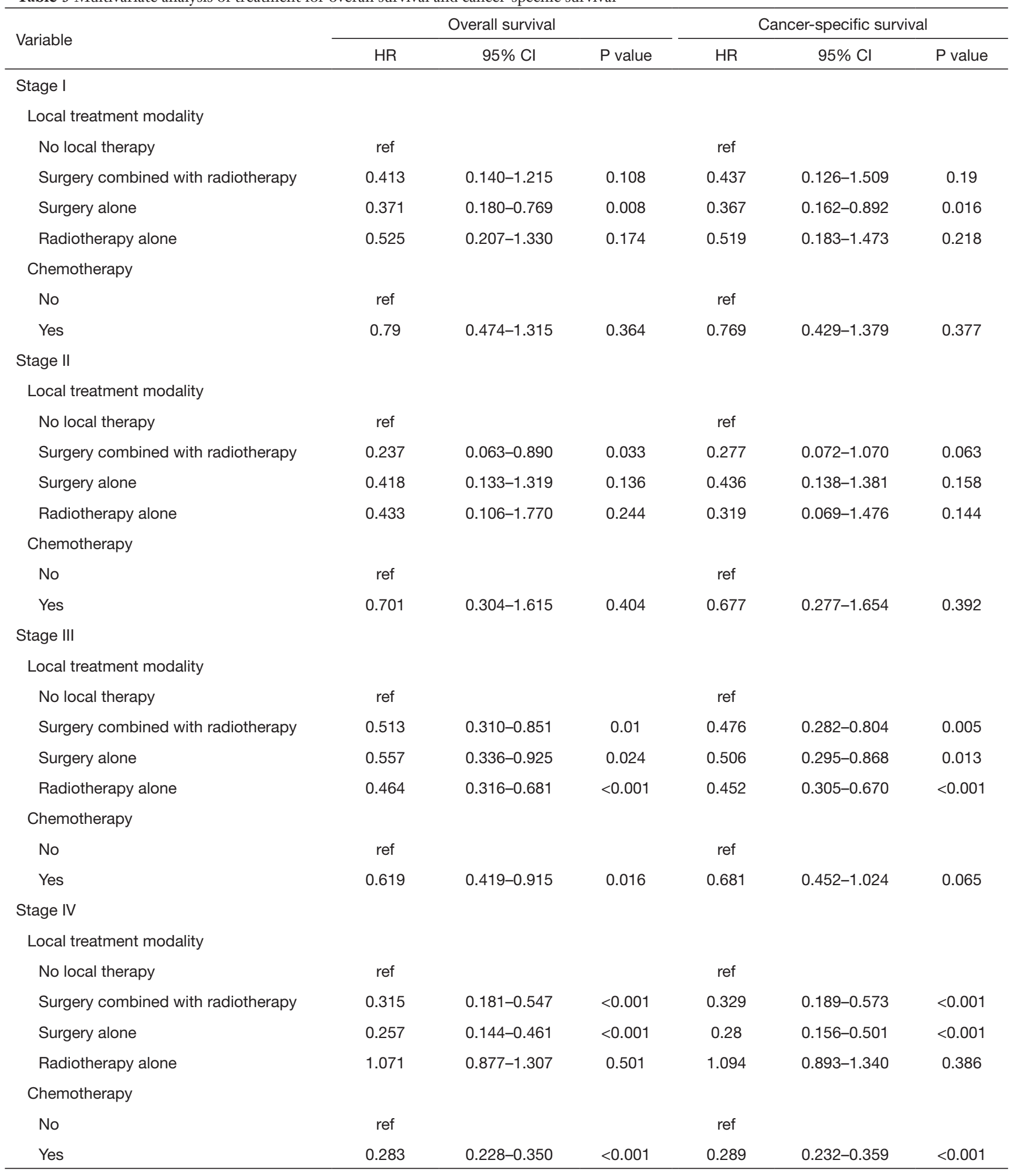


longer in the surgery group than in the non-surgery group, even for stage IV patients. Although the proportion of patients suitable for surgery was small, choosing the right patients for surgery could improve the prognosis.

Earlier studies mostly focused on surgery in patients with early stage C-SCLC. In their retrospective study, Babakoohi et al. reported that the OS of C-SCLC was longer than pure SCLC in the surgery group (15 vs. 10.8 months); however, there was no difference in the non-surgery group (17). Men et al. found that for C-SCLC, the 5-year OS rate in the surgery group was higher than in the non-surgery group (48.9\% vs. 36.6\%) (4). Therefore, surgery might be more significant for C-SCLC patients compared with SCLC patients. The surgical indication for C-SCLC should be broader than pure-SCLC. It will be interesting for future studies to investigate patients who would be suitable for surgery.

Studies on radiotherapy in C-SCLC are limited. This study showed that stage I and II patients were less likely to receive radiotherapy alone as a local treatment and only occasionally received it combined with surgery. Radiotherapy alone was not an independent factor of prognosis for patients with stage I and II disease, but surgery combined with radiotherapy significantly improved OS for patients with stage II. Stage III patients were more often treated with radiotherapy. Either radiotherapy alone or combined with surgery could significantly improve the OS and CSS for stage III patients. The data showed that it was not an independent prognostic factor, although only $40.6 \%$ of the stage IV patients underwent radiotherapy alone as local therapy. Surgery combined with radiotherapy could significantly improve the OS and CSS for patients with stage IV. A previous study reported that postoperative radiotherapy (PORT) was a significant prognostic factor for C-SCLC patients with pathological N2 stage or a proportion of metastatic lymph nodes greater than $10 \%(18)$. Further studies are needed to investigate the value of radiotherapy for C-SCLC.

Our data showed that chemotherapy was widely used in patients with C-SCLC. However, chemotherapy was only an independent predictor of an improved survival in patients with stage III and IV. Men et al. reported that even though nearly $84 \%$ of C-SCLC patients received chemotherapy, there were no significant differences in the 5 -year survival rates between the chemotherapy group and the non-chemotherapy groups $(37.7 \%, 35.4 \%)$ (4). Previous studies also found that the response rate of chemotherapy regimens for pure SCLC was lower when it was applied in the treatment of C-SCLC (15). These findings equally indicated a challenge for chemotherapy in the treatment of C-SCLC. Thus, the difficulty stems from optimizing the indications in the clinical practice and determining the regimens and combined treatment modalities for the administration of chemotherapy in C-SCLC.

To our knowledge, this is the first large-sample retrospective study to summarize the clinical characteristics, prognosis, and treatment of C-SCLC. However, there were several limitations to this study. Firstly, it is retrospective; as a result, there are inevitable selection biases, information biases, and confounding biases. Secondly, the study did not include all possible prognostic factors for analysis, especially the newly discovered factors, such as NLR, CRP, and NSCLC components, due to the characteristics of the database. Finally, as this study analyzed data from over 12 years, changes in diagnostic and therapeutic techniques during this timeframe may affect the reliability of conclusions.

\section{Conclusions}

In summary, our study proved that the significant independent determinants of an improved survival rate included being younger than 65 years, female, and having an earlier stage at presentation. Surgery may significantly improve the survival outcomes of patients with any stage of C-SCLC. Surgery combined with radiotherapy is associated with an increased OS in patients with stage II, and radiotherapy alone is associated with an increased OS and DSS in patients with stage III. Chemotherapy is only an independent predictor for the survival of patients with stages III and IV. These findings may help to determine the treatments used and further research on this rare disease.

\section{Acknowledgments}

Funding: None.

\section{Footnote}

Reporting Checklist: The authors have completed the STROBE reporting checklist. Available at http://dx.doi. org/10.21037/tcr-20-968

Data Availability: The datasets used and/or analyzed during the current study are available from the corresponding author on reasonable request. 
Conflicts of Interest: All authors have completed the ICMJE uniform disclosure form (available at http://dx.doi. org/10.21037/tcr-20-968). The authors have no conflicts of interest to declare.

Ethical Statement: The authors are accountable for all aspects of the work in ensuring that questions related to the accuracy or integrity of any part of the work are appropriately investigated and resolved.

Open Access Statement: This is an Open Access article distributed in accordance with the Creative Commons Attribution-NonCommercial-NoDerivs 4.0 International License (CC BY-NC-ND 4.0), which permits the noncommercial replication and distribution of the article with the strict proviso that no changes or edits are made and the original work is properly cited (including links to both the formal publication through the relevant DOI and the license). See: https://creativecommons.org/licenses/by-nc-nd/4.0/.

\section{References}

1. Zhang C, Yang H, Zhao H, et al. Clinical outcomes of surgically resected combined small cell lung cancer: a twoinstitutional experience. J Thorac Dis 2017;9:151-8.

2. Woo JH, Kim MY, Lee KS, et al. Resected Pure Small Cell Lung Carcinomas and Combined Small Cell Lung Carcinomas: Histopathology Features, Imaging Features, and Prognoses. AJR Am J Roentgenol 2019;212:773-81.

3. Zhao X, McCutcheon JN, Kallakury B, et al. Combined Small Cell Carcinoma of the Lung: Is It a Single Entity? J Thorac Oncol 2018;13:237-45.

4. Men Y, Hui Z, Liang J, et al. Further understanding of an uncommon disease of combined small cell lung cancer: clinical features and prognostic factors of 114 cases. Chin J Cancer Res 2016;28:486-94.

5. Murase T, Takino H, Shimizu S, et al. Clonality analysis of different histological components in combined small cell and non-small cell carcinoma of the lung. Hum Pathol 2003;34:1178-84.

6. Fukui T, Tsuta K, Furuta K, et al. Epidermal growth factor receptor mutation status and clinicopathological features of combined small cell carcinoma with adenocarcinoma of the lung. Cancer Sci 2007;98:1714-9.

7. Calbo J, van Montfort E, Proost N, et al. A functional role for tumor cell heterogeneity in a mouse model of small cell lung cancer. Cancer Cell 2011;19:244-56.
8. Hahn EE, Gould MK. Lung Cancer Screening and Smoking Cessation: Never Too Early or Too Late. J Natl Cancer Inst 2018;110:1157-58.

9. Ito T, Kudoh S, Ichimura T, et al. Small cell lung cancer, an epithelial to mesenchymal transition (EMT)-like cancer: significance of inactive Notch signaling and expression of achaete-scute complex homologue 1. Hum Cell 2017;30:1-10.

10. Hassan WA, Takebayashi SI, Abdalla MOA, et al. Correlation between histone acetylation and expression of Notch1 in human lung carcinoma and its possible role in combined small-cell lung carcinoma. Lab Invest 2017;97:913-21.

11. Meder L, Konig K, Ozretic L, et al. NOTCH, ASCL1, p53 and RB alterations define an alternative pathway driving neuroendocrine and small cell lung carcinomas. Int J Cancer 2016;138:927-38.

12. Asahina M, Fukumura $Y$, Mamat $\mathrm{O}$, et al. A case of combined small cell lung carcinoma with unique morphology: Investigation of tumorigenesis. Pathol Int 2018;68:618-23.

13. Shao N, Cai Q. High pretreatment neutrophil-lymphocyte ratio predicts recurrence and poor prognosis for combined small cell lung cancer. Clin Transl Oncol 2015;17:772-8.

14. Moniodis A, Racila E, Divo M. Case report: combined small cell lung cancer in a lung transplant recipient. Transplant Proc 2015;47:852-4.

15. Wang X, Jiang R, Li K. Prognostic significance of pretreatment laboratory parameters in combined small-cell lung cancer. Cell Biochem Biophys 2014;69:633-40.

16. National Comprehensive Cancer Network. NCCN Clinical Practice Guidelines in Oncology: Small Cell Lung Cancer (2019 V1). Washington: National Comprehensive Cancer Network; 2018 [cited 2018 Oct 10]. Available online: https://www.nccn.org/professionals/physician_gls/ default.aspx\#sclc

17. Babakoohi S, Fu P, Yang M, et al. Combined SCLC clinical and pathologic characteristics. Clin Lung Cancer 2013;14:113-9.

18. Men Y, Luo Y, Zhai Y, et al. The role of postoperative radiotherapy (PORT) in combined small cell lung cancer (C-SCLC). Oncotarget 2017;8:48922-29.

Cite this article as: $\mathrm{Xu} \mathrm{L}$, Zhang G, Song S, Zheng Z. Prognostic factors, treatment, and outcomes in combined small cell lung cancer: a SEER survey from 2004 to 2015. Transl Cancer Res 2020;9(9):5292-5303. doi: 10.21037/tcr-20-968 